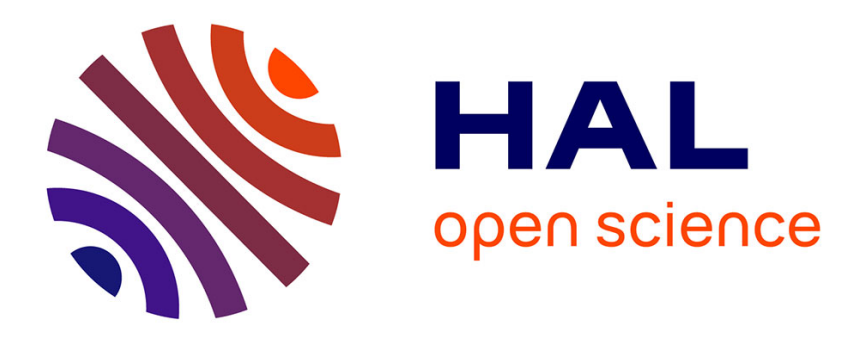

\title{
Constant speed optimal reciprocal collision avoidance
}

Nicolas Durand

\section{To cite this version:}

Nicolas Durand. Constant speed optimal reciprocal collision avoidance. Transportation research. Part

C, Emerging technologies, 2018, pp.Pages 366-379. 10.1016/j.trc.2018.10.004 hal-01903018

\section{HAL Id: hal-01903018 \\ https: / hal-enac.archives-ouvertes.fr/hal-01903018}

Submitted on 26 Oct 2018

HAL is a multi-disciplinary open access archive for the deposit and dissemination of scientific research documents, whether they are published or not. The documents may come from teaching and research institutions in France or abroad, or from public or private research centers.
L'archive ouverte pluridisciplinaire HAL, est destinée au dépôt et à la diffusion de documents scientifiques de niveau recherche, publiés ou non, émanant des établissements d'enseignement et de recherche français ou étrangers, des laboratoires publics ou privés. 


\title{
Constant Speed Optimal Reciprocal Collision Avoidance
}

\author{
Nicolas Durand \\ ENAC \\ nicolas.durand@enac.fr
}

\begin{abstract}
In this article, the Optimal Reciprocal Collision Avoidance (ORCA) algorithm is modified to make it work for speed constrained aircraft. The adaptation of ORCA to aircraft conflict resolution shows that when the speed norm is constrained, aircraft flying within the same speed range with small angle converging trajectories tend to remain on parallel tracks, preventing a resolution of the conflict. The ORCA algorithm is slightly modified to avoid this behavior. In the new algorithm called CSORCA (Constant Speed Optimal Reciprocal Collision Avoidance), the directions of the semi-plane used to calculate the conflict free maneuvers are modified when the relative speed vector is in the semi-circular part of the conflicting area. After explaining the reasons that make the original algorithm fail in the constant speed environment, the modification made on the algorithm is detailed and its impact on a simple example is shown. The new strategy is also compared to an Add-Up strategy close to the Airborne Separation Assurance System (ASAS) strategy found in the literature. Hundreds of fast time simulations are then performed to compare the two versions of the algorithm for different traffic densities in the horizontal plane. In these simulations the speed norm is first constrained. The aircraft can only change direction with a limited turning rate. Simulations with released speed constraints are then performed to compare the behavior of both algorithms in a more general environment. In all the scenarios tested, CSORCA is more efficient than ORCA to solve conflicts. Keywords: ORCA, CSORCA, self-separation, geometrical algorithm, air traffic
\end{abstract}


control, UAS 


\section{List of Figures}

$1 \quad$ Conflicting aircraft model: a conflict will occur within time $\tau$ if and only if the relative speed $\overrightarrow{v_{r}}$ lies in the forbidden zone in red. 12

2 Conflicting aircraft model: the effort is shared by the two aircraft. The new speeds must be chosen on the green arc. . . . . . . . . . 12

3 Multi-Conflicting aircraft model: a conflict will occur within time $\tau$ if and only if the relative speed $\overrightarrow{v_{r}}$ lies in the forbidden zone in red. . . . . . . . . . . . . . . . . . . . . . 13

4 Conflicting aircraft model: in this example $\vec{c}$ (the projection of $\overrightarrow{v_{r}}$ outside the forbidden zone) is on the circular part of the red zone. . . . . . . . . . . . . . . . . . . 16

5 Two aircraft conflict with the original ORCA algorithm. Aircraft are turned opposite directions and fly parallel tracks without reaching their destinations. . . . . . . . . . . . . . . . . . . . 17

6 New model: the relative speed $\overrightarrow{v_{r}}$ is now projected on the closest side of the cone instead of the circular part. . . . . . . . . . . . . 18

7 Two aircraft conflict with the modified ORCA algorithm. Both aircraft are turned left and the conflict is easily solved. . . . . . . 19

8 Ten aircraft conflict with the original ORCA algorithm. Aircraft choose parallel tracks. . . . . . . . . . . . . . . . . . . . . . . . . 19

$9 \quad$ Ten aircraft conflict with the modified ORCA algorithm. Every aircraft reaches its destination without conflict. . . . . . . . . . . 20

$10 \quad 8$ aircraft conflict with the Add-Up Strategy. . . . . . . . . . . . 21

11 A scenario example with 10 aircraft and no speed norm modification. 22

12 A scenario example with 130 aircraft and 30\% speed norm modification. . . . . . . . . . . . . . . . 23 


\section{List of Tables}

1 Total number of failures, due to border violations or remaining conflicts, mean and max delays for 10 to 90 aircraft with no speed norm variation and 3 degrees/second maximum turning rate. Left: CSORCA - Right: ORCA . . . . . . . . . . . . . . . . 24

2 Total number of failures, for different values of $\tau$ for 10 to 90 aircraft with no speed norm variation and 3 degrees/second maximum turning rate. CSORCA . . . . . . . . . . . . . . . . . 25

3 Add-Up Strategy: Total number of failures, due to border violations or remaining conflicts, mean and max delays for 10 to 90 aircraft with no speed norm variation and 3 degrees/second maximum turning rate. . . . . . . . . . . . . . . . . . . 26

4 Total number of failures, due to border violations or remaining conflicts, mean and max delays for 10 to 90 aircraft with no speed norm variation and 6 degrees/second maximum turning rate. Left: CSORCA - Right: ORCA . . . . . . . . . . . . . . . . 27

5 Total number of failures, due to border violations or remaining conflicts, mean and max delays for 10 to 90 aircraft with $+-5 \%$ maximum speed norm variation and 3 degrees/second maximum turning rate. Left: CSORCA - Right: ORCA . . . . . . . . . . . 28

6 Total number of failures, due to border violations or remaining conflicts, mean and max delays for 10 to 90 aircraft with no $+-15 \%$ maximum speed norm variation and 3 degrees/second maximum turning rate. Left: CSORCA - Right: ORCA . . . . . 29 


\section{Introduction}

The growing traffic of Unmanned Airborne Systems (UAS) flying in the lower airspace is leading researchers to study self-separation algorithms in order to separate UAS. Self-separation has been widely studied in the case of two

5 UAS Durand et al. (2000); Zeghal (1998); Eby and Kelly (1999); Hoekstra et al. (2002), but dealing with complex situations involving many aircraft (more than 2) has mainly been treated by adding pairwise maneuvers.

In this article, the Optimal Reciprocal Collision Avoidance (ORCA) algorithm van den Berg et al. (2011) is modified to improve its performance for speed constrained aircraft. The modification is tested on different densities in order to show that it improves the performance of the algorithm when the speed norms are constrained without decreasing its performance when speed constraints are relaxed.

Van den Berg et al. proposed an Optimal Reciprocal Collision Avoidance 15 (ORCA) Algorithm van den Berg et al. (2011) using a simple geometric approach in order to guarantee conflict resolution for the next $\tau$ minutes in an autonomous manner. Each agent is only aware of the surrounding traffic. For multiple conflict situations, the algorithm finds compatible maneuvers that separate aircraft in complex situations in a decentralized way without sequencing maneuvers. This makes it very different from previous decentralized approaches such as sliding force:Zeghal (1998) or ASAS Hoekstra et al. (2002) that simply add up the pairwise maneuvers when dealing with multiple aircraft conflicts.

a It was adapted to aircraft separation by Snape et al. in 2010 Snape and Manocha (2010). The algorithm guarantees a conflict free solution for the next

${ }_{25} \tau$ minutes if the agents can change their speed without constraint. In the worst case, when the density gets too high, some agents may completely be stopped before heading back to their destination.

The ORCA algorithm was tested in a previous project Durand and Barnier (2015) and showed some limits when the speed norm was constrained. We 30 showed that when speed norms are similar and strongly constrained, the ORCA 
algorithm cannot handle low densities of traffic. Aircraft converging on a point with small angles tend to choose parallel trajectories that do not solve the conflicts but divert them far from their destination. This phenomenon can be avoided by slightly modifying the ORCA algorithm. This modification and its

35 consequences on a series of tests involving different densities of traffic is the main contribution of this article.

\subsection{Related Works}

Aircraft conflict resolution is still performed by humans in a centralized approach. Air traffic controllers have a global view of the whole situation and give maneuvers to aircraft to keep a minimum vertical and horizontal separation between them. In the 90s, we showed that conflict resolution was highly combinatorial and could not be globally solved with local optimization methods Durand et al. (1996). We proposed a genetic algorithm to solve multiple aircraft conflicts using a centralized approach with simple maneuvers (similar to

45 those used by air traffic controllers) and showed that we were able to solve every conflict on real traffic data Durand and Alliot (1997).

Centralized methods can be divided into two main categories. Some methods Krella et al. (1989); Chiang et al. (1997); Hu et al. (2002) use greedy sequential algorithms to optimize trajectories one by one after ranking the aircraft. However, finding an appropriate ordering is challenging Archambault and Durand (2004). The others, mentioned in the next paragraph, try to find the global optimum without the need to prioritize aircraft.

Using evolutionary computation, our team was the first to address conflict resolution globally Durand et al. (1996). Others later introduced a powerful 55 Semidefinite Programming approach Oh et al. (1997); Frazzoli et al. (2001), which is also able to handle multiple aircraft in a single scenario. However the solution is only locally optimal and the model requires constant speeds and perfect trajectory prediction. In the early 2000s, a mathematical model using Mixed Integer Linear Programming, which could be solved by CPLEX and ensured the global optimality of the solution, was proposed Pallottino et al. 
(2001, 2002). The model was extended in 3D in 2008, but required constant speed during climbing phases Christodoulou and Kontogeorgou (2008). Uncertainties on the trajectory heading were added to the horizontal model in 2009, but all maneuvers still needed to be executed at the same time (at every optimization step) Gariel and Feron (2009). The method is powerful but cannot be used for developing a realistic advisory tool for controllers.

More recently, in the horizontal plane, Omer et al. Omer and Farges (2013) proposed a Mixed-Integer Linear Programming approach that takes into account the trajectory recovery. Alonso-Ayuso et al. Alonso-Ayuso et al. (2016) proposed a Mixed Integer Non Linear Optimization model to deal with the horizontal fase and take the trajectory recovery into account. Peyronne et al. Peyronne et al. (2015) introduce a trajectory model using B-Splines and a semi-infinite programming formulation of the constraints. The combinatorial aspect of the resolution of multi-aircraft conflicts is solved with a genetic algorithm like in

75 Durand et al. (1996). Rey et al. Rey and Hijazi (2017) recently proposed a new complex number formulation and convex relaxations for the centralized problem and showed that it could significantly reduce the resolution time.

In 2013, our team used a Constraint Programming approach to globally solve complex conflicts Allignol et al. (2013). For each aircraft, a number of alternative trajectories and a matrix of pairwise conflicts were precalculated, taking various uncertainties into account, before the optimization process was performed. This approach separated the problem model from its resolution and was close to the graph model proposed by Lehouillier et al. (2017).

In the 1990s, a wide debate started among the Air Traffic Control communities on the possibility of eliminating centralized control systems by developing autonomous algorithms for solving conflicts. At that time the motivation was to reduce the cost of air traffic control. The recent perspective of development of Unmanned Airborne Systems in the lower airspace gives a new interest to autonomous approaches and the high traffic demand in the lower airspace Bulusu

90 et al. (2016) will create complex situations that centralized approaches will probably not be able to handle. 
The first effective approach used sliding forces to coordinate maneuvers between aircraft Zeghal (1998). Potential or vortex fields Košecká et al. (1998) as well as a model based on an analogy with electrical particle repulsion Eby and 95 Kelly (1999) were also used. These algorithms took into account an important constraint of aircraft: the speed constraint. During a level flight an aircraft can easily change direction respecting a maximum turning rate but it can barely modify its speed range. For situations involving many aircraft, these three algorithms relied on the principle that forces or potential fields virtually generated by aircraft on each other would add up. There is no guarantee that this principle always leads to a conflict free solution. Eby at al.'s approach Eby and Kelly (1999) inspired the Airborne Separation Assurance System (ASAS) approach developed by Hoekstra et al. Hoekstra et al. (2002). ASAS uses a Modified Voltage Potential (MVP) close to Eby's definition. It was tested in different contexts such as the Mediterranean Free-Flight Ruigrok and Hoekstra (2007). More recently, it was used to model airspace stability and capacity in a decentralized separation context Sunil et al. (2017) and in a Capacity Assessment Model Emmanuel Sunil and Hoekstra (2018).

A geometric optimization approach was proposed by Bilimoria Bilimoria (2000) in 2000 and used in the Future Air traffic management concepts Evaluation Tool (FACET) project D. Bilimoria et al. (2001), but it solved complex situations by using an iterative process. This is also the case of the Free-flight Autonomous and Coordinated Embarked Solver Granger et al. (2001b a) proposed by Granger et al. in 1999 that used a token allocation strategy to coordinate sequential maneuvers.

In 2007, Wang et al. Hwang et al. (2007) introduced a geometric approach that required a shared knowledge of the whole situation. This strong hypothesis undermines autonomous conflict resolution. In an autonomous context, aircraft are only aware of their surrounding context. In contrast, implementing the same central approach in every aircraft leads to a non autonomous resolution. Leny et al. Le Ny and Pappas (2010) in 2010 also used a geometric approach for scheduling crossing times of aircraft through a metering fix. The model was not 
adapted to general situations. Pallottino et al. Pallottino et al. (2007) proposed a model where coordination is ensured by protected stacking areas in which each aircraft can move without conflicting with other aircraft. The areas are headed to the flight destination. Even if the aircraft is flying at constant speed these protected areas can stop moving and ensure that a solution can be found even in high densities. Schouwenaars et al. Schouwenaars and Feron (2004) used the same kind of approach to plan safe trajectories in a decentralized way. In 2009, Alam et al. Alam et al. (2009) proposed to use data mining to determine which conflict detection algorithm is more likely to give an accurate answer depending on the traffic situation.

Geometric approaches using automated coordination were first introduced by Van den Berg et al. van den Berg et al. (2008), first with the Reciprocal

135 Velocity Obstacles and then with the Optimal Reciprocal Collision Avoidance (ORCA) van den Berg et al. (2011) algorithm which is used in the present paper. ORCA was first meant to be used to simulate robots using self-separation logic in a $2 \mathrm{D}$ environment. In air traffic control, Snape et al. Snape and Manocha (2010) have extended the model to the vertical dimension without segregating the horizontal and vertical maneuvers. More recently, Geometric approaches have led to the definition of Solution Space Diagrams that were used to analyze the dynamic of air traffic controller workload d'Engelbronner et al. (2015) and were recently compared to the Modified Voltage Potential approach Balasooriyan (2017) showing that the latter seemed to be more efficient in many situations.

\subsection{Outline}

Section 2 presents the geometrical algorithm and describes the problem that occurs when two similar speed aircraft which can only change headings converge with a small angle. In section 3 , the modification made to the algorithm is detailed and its consequence is shown on simple examples. The new algorithm is also compared to a maneuver Add-Up strategy for multiple aircraft conflicts close to the ASAS multiple aircraft conflict strategy. Section 4 describes the simulation scenarios used to validate the modification on different traffic densities. 
The Add-Up strategy is used to compare the results. Finally the speed norm constraint is relaxed in order to check the behavior of the algorithm for high traffic densities. A conclusion and perspectives are given in section 5

\section{Detect and Avoid Model}

This section describes the Optimal Reciprocal Collision Avoidance (ORCA) algorithm developed in van den Berg et al. (2011) and its adaptation to the case where speed norms are constrained. First the maneuver model is detailed for an aircraft pair, then the maneuver calculation is explained when more than two aircraft are involved in a conflict.

\subsection{Constraint Model}

Let $d$ be the target separation distance and $\tau$ be a look ahead time. In figure 1, let us consider aircraft $A$ and aircraft $B$. Aircraft $B$ 's position can be represented in the referential of aircraft $A$. Consider a circle of radius $d$ centered at aircraft $B$, the two lines issued from position $A$, tangent to the circle of radius $d$ form a cone. If the relative speed $\overrightarrow{v_{r}}=\overrightarrow{v_{A}}-\overrightarrow{v_{B}}$ lies in this cone, a conflict will occur in the future. A circle of size $\frac{d}{\tau}$ centered at $B^{\prime}$ such that $\overrightarrow{A B^{\prime}}=\frac{\overrightarrow{A B}}{\tau}$ defines a zone (in light red) bounded by the bold line in figure $1 . \overrightarrow{v_{r}}$ lies in this 170 zone if and only if it intersects the disc of size $\frac{d}{\tau}$ centered in $B^{\prime}$, i.e. if and only if there exists $\lambda \in\left[0: 1\left[\right.\right.$ such that $\left|\lambda \overrightarrow{v_{r}}-\overrightarrow{A B^{\prime}}\right|<\frac{d}{\tau}$ or $\left|\lambda \tau \overrightarrow{v_{r}}-\overrightarrow{A B}\right|<d$ which means that $\mathrm{A}$ and $\mathrm{B}$ are in conflict at time $\lambda \tau<\tau$. Consequently a conflict will occur within time $\tau$ if and only if $\overrightarrow{v_{r}}$ lies in this light red zone.

If this is the case, then it is necessary to modify speed vectors $\overrightarrow{v_{A}}$ and $\overrightarrow{v_{B}}$ in 175 such a way that the resulting relative speed vector $\overrightarrow{v_{r}^{\prime}}$ is outside of the constraint. Let $\vec{c}$ be the projection of $\overrightarrow{v_{r}}$ on the closest edge of the red zone.

The ORCA algorithm is based on the principle that the effort to keep $\overrightarrow{v_{r}}$ out of the red area should be minimal and shared by the two aircraft. In its original version the necessary minimal speed modification $\vec{c}$ is shared equally between the two aircraft and defines two semi-planes $P_{B \rightarrow A}$ and $P_{A \rightarrow B}$ (in light 
green) perpendicular to vector $\vec{c}$ in which aircraft $A$ and $B$ can choose their new speeds.

If the modified speeds $\overrightarrow{v_{A}^{\prime}}$ and $\overrightarrow{v_{B}^{\prime}}$ are in these semi-planes, the new relative speed $\overrightarrow{v_{r}^{\prime}}$ is out of the red zone and the conflict is solved.

Indeed, the semi-plane corresponding to the modified speed of aircraft $A$ is defined by the equation:

$$
\left(\overrightarrow{v_{A}^{\prime}}-\left(\overrightarrow{v_{A}}+\frac{\vec{c}}{2}\right)\right) \cdot \vec{c} \geq 0
$$

The semi-plane corresponding to the modified speed of aircraft $B$ is defined by the equation:

$$
\left(\overrightarrow{v_{B}^{\prime}}-\left(\overrightarrow{v_{B}}-\frac{\vec{c}}{2}\right)\right) \cdot \vec{c} \leq 0
$$

The difference of the two previous equations leads to:

$$
\left(\overrightarrow{v_{r}^{\prime}}-\left(\overrightarrow{v_{r}}+\vec{c}\right)\right) \cdot \vec{c} \geq 0
$$

This equation guarantees that $\overrightarrow{v_{r}^{\prime}}$ is outside the truncated cone.

When three or more aircraft are in conflict, each aircraft pair involving aircraft $A$ defines a semi-plane for aircraft $A$ (see figure 3 ) and the new speed for $A$ is chosen in the intersection of the semi-planes generated by every other aircraft. If the intersection of the semi-planes is empty, every semi-plane is equally slightly moved until the intersection exists. This process is described by Van den Berg van den Berg et al. (2011).

This is the main difference with the ASAS approach based on Modified Potential Voltage (MVP). In the (MVP) approach, the maneuvers are calculated for pairs of aircraft and when 2 or more maneuvers are calculated for the same aircraft, the resulting maneuver is the sum of each single maneuver.

\subsection{Possible Heading Range}

In this paper, heading changes are the only possible maneuvers for aircraft. The capacity of aircraft to change speed is small. A velocity increase or reduction 


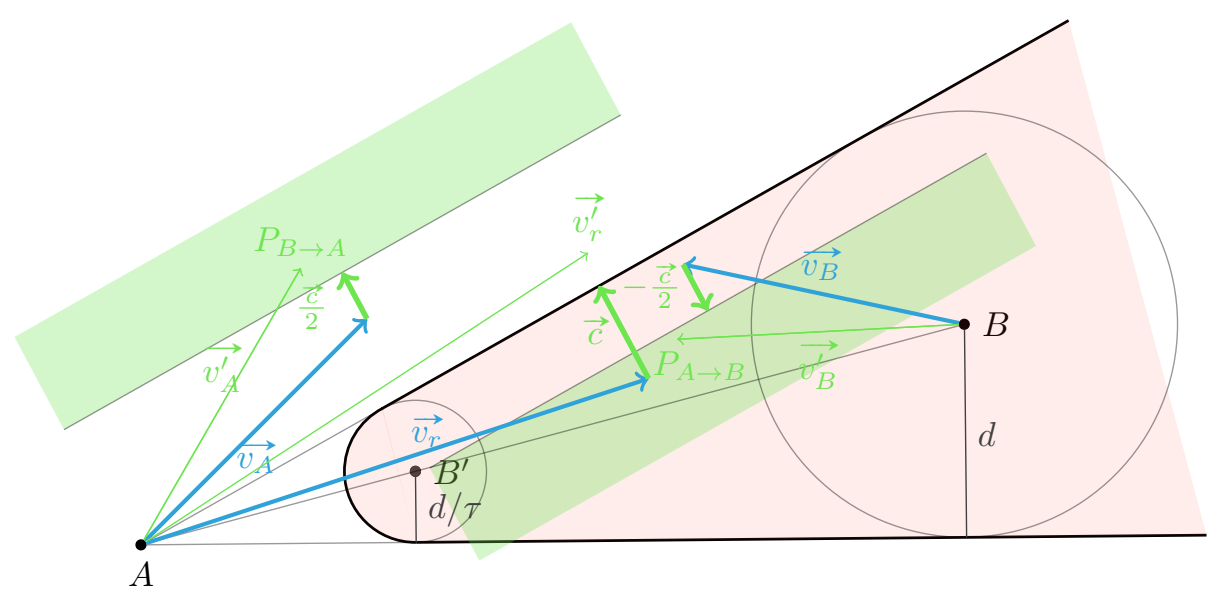

Figure 1: Conflicting aircraft model: a conflict will occur within time $\tau$ if and only if the relative speed $\overrightarrow{v_{r}}$ lies in the forbidden zone in red.

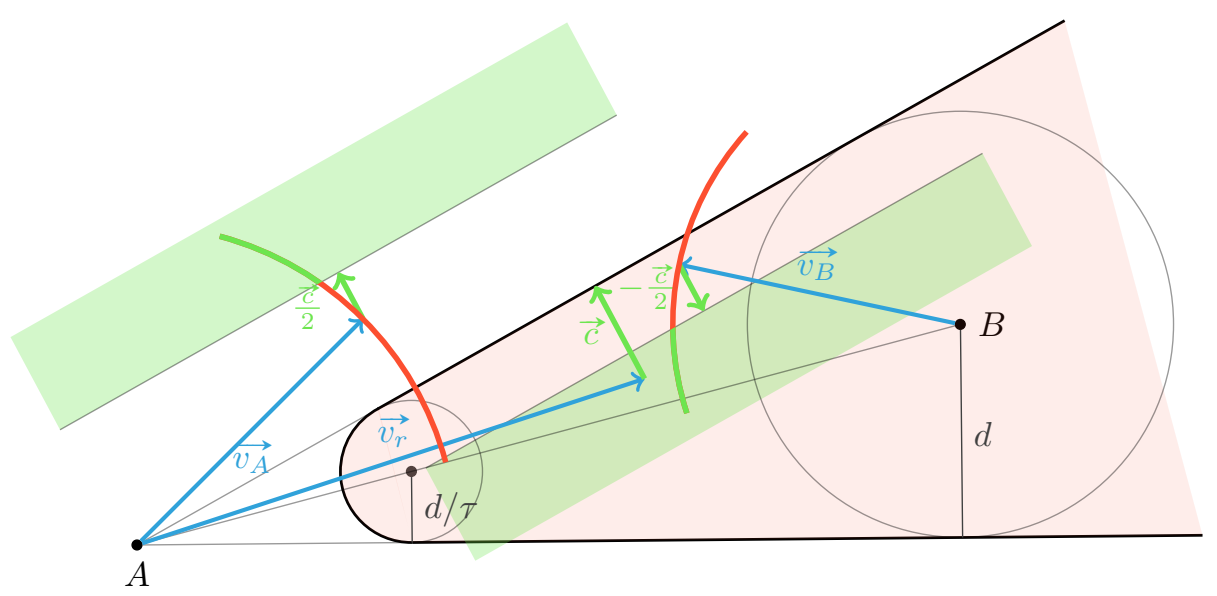

Figure 2: Conflicting aircraft model: the effort is shared by the two aircraft. The new speeds must be chosen on the green arc. 


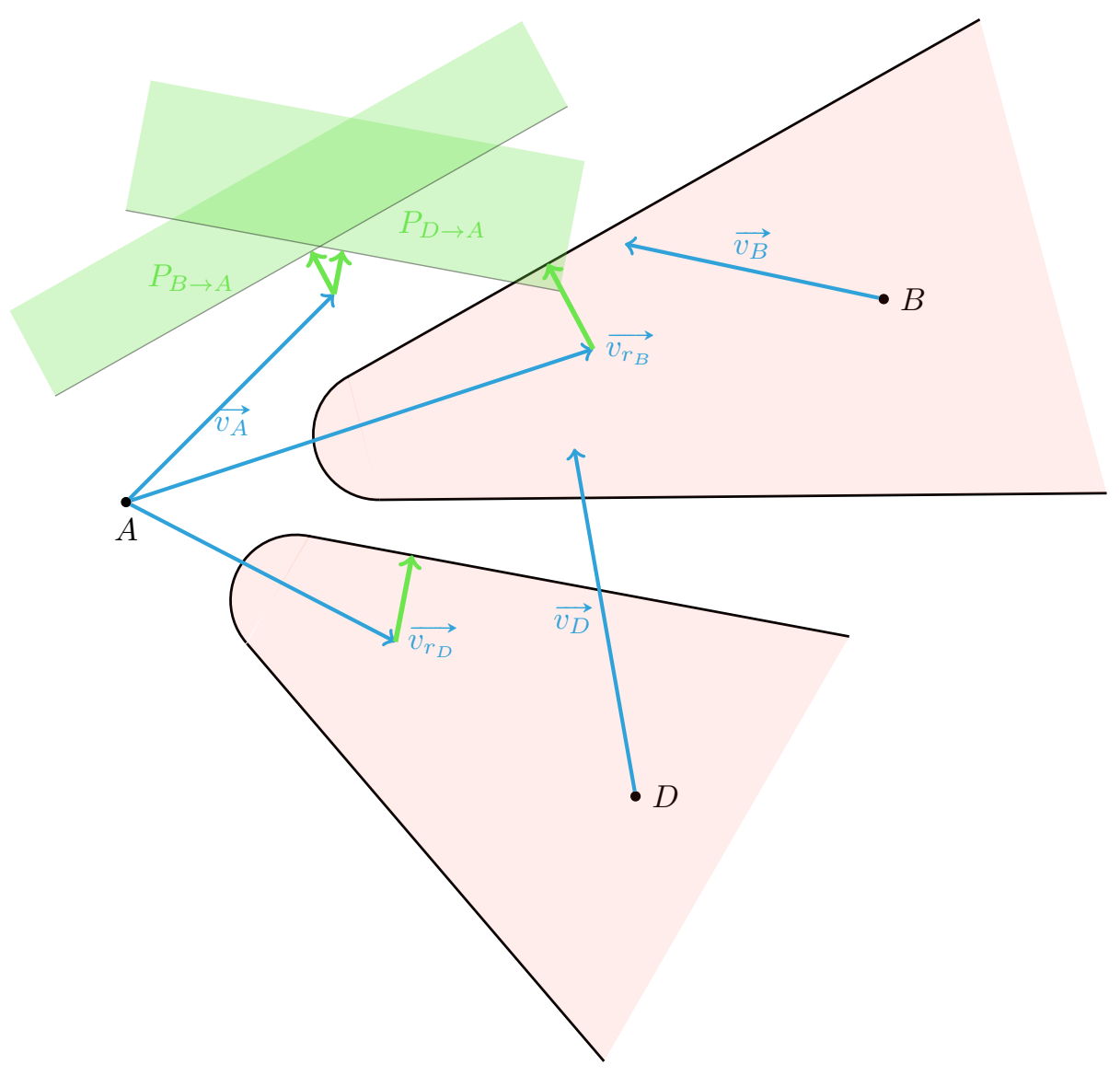

Figure 3: Multi-Conflicting aircraft model: a conflict will occur within time $\tau$ if and only if the relative speed $\overrightarrow{v_{r}}$ lies in the forbidden zone in red. 
is limited in amplitude and takes time. In the ERASMUS speed control project,

the speed modification range was set to $[-6 \% ;+3 \%]$ Bonini et al. $(2009)$.

The norm of the aircraft speeds remains constant throughout the conflict resolution process if and only if:

$$
\begin{aligned}
& \left\|\overrightarrow{v_{A}^{\prime}}\right\|=\left\|\overrightarrow{v_{A}}\right\| \\
& \left\|\overrightarrow{v_{B}^{\prime}}\right\|=\left\|\overrightarrow{v_{B}}\right\|
\end{aligned}
$$

Because of the speed norm constraint, the new vectors must be chosen on the green arc (see figure 2). The arc length is limited by the maximum turning rate. In the further experiments, we compare a standard turning rate (3 degrees per second) and a doubled standard turning rate (6 degrees per second). If the time step is set to 3 seconds, the arc range is \pm 9 degrees for the standard turning rate and \pm 18 degrees for the doubled standard turning rate.

215

Aircraft can limit the speed change by choosing the new vectors that give the smallest heading change. This strategy was called "closest" by Allignol et al. Allignol et al. (2017) because the current heading $h_{c}$ is modified as little as possible.

In the present case, in order to ensure that aircraft reach their destinations, the ideal heading $h_{i}$ (the heading toward the destination) is first calculated and the "closest" approach is used considering $h_{i}$ instead of the current heading $h_{c}$

Algorithm 1 describes the ORCA algorithm. Time is discretized into time steps $t_{s}$ (for the experiments $t_{s}=3$ seconds). As long as every aircraft has not reached its destination, every aircraft pair $(i, j)$ is checked to calculate semi-planes $P_{j \rightarrow i}$ and $P_{i \rightarrow j}$. For every aircraft $i$, the intersection of the $P_{k \rightarrow i}$ with $k \neq i$ semi-planes creates a convex constraint surface $C_{i}$. The intersection of $C_{i}$ and the arc $A_{i}$ of possible aircraft $i$ turning range defines the arc $S_{i}$ of possible conflict free headings. The new current heading $h_{c}$ is then chosen as close as possible to the ideal heading $h_{i}$ (facing the destination). If the intersection of the semi-planes is empty, every semi-plane is equally slightly moved until an intersection appears. The convex $C_{i}$ does not guarantee a conflict free maneuver anymore, but it remains close to the conflict free domain. 


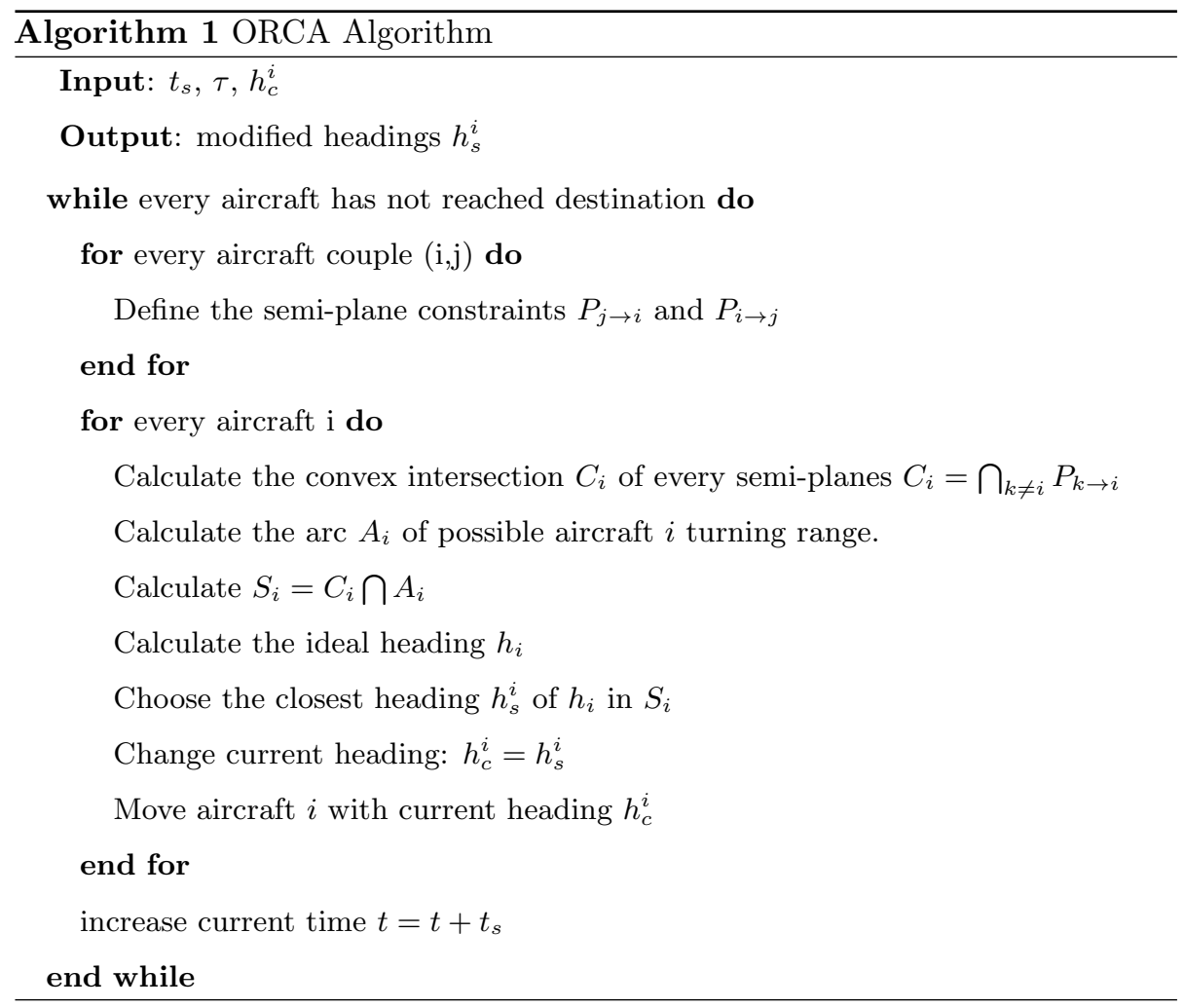

\section{ORCA modification for Constant Speeds}

The ORCA algorithm described in the previous section does not solve conflicts

for small angle converging aircraft that can only change headings Durand and Barnier (2015). It tends to postpone the conflict outside of time window $\tau$.

\subsection{Critical cases}

Figure 4 represents another conflict in which the relative speed is close to the circular part of the cone. When the relative speed vector $\overrightarrow{v_{r}}$ is close to the circular part of the cone, the direction of vector $\vec{c}$ moves the corresponding semi-planes for $\overrightarrow{v_{A}}$ and $\overrightarrow{v_{B}}$ in directions that are not parallel to one of the cone sides (see figure 4. As a result, depending on the initial conditions, the new aircraft speeds tend to become parallel instead of choosing directions that solve the conflict. 


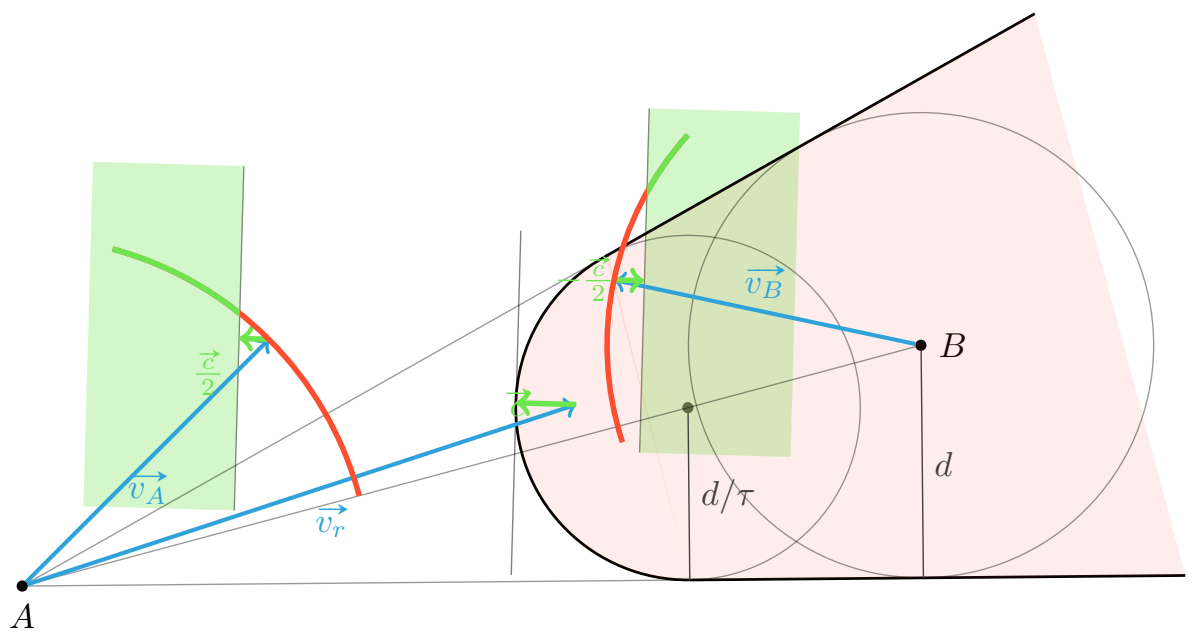

Figure 4: Conflicting aircraft model: in this example $\vec{c}$ (the projection of $\overrightarrow{v_{r}}$ outside the forbidden zone) is on the circular part of the red zone.

This is called the horizon effect in game theory. Instead of solving the conflict, the algorithm tends to postpone the conflict outside the time window.

When the relative vector is in the circular part of the forbidden cone, it means that the conflict is "just starting" and it is "cheaper" to postpone it than to solve it. This continuous approach of solving the conflict prevents both aircraft from making a resolution decision. Aircraft tend to end-up parallel and divert from their destination. This phenomenon is made worse by the speed norm constraint. In figure 5, the two aircraft converging to the center of the figure (red lines) with the same speed are turned in opposite directions and progressively choose parallel speeds. The conflict is solved, because the aircraft are parallel, but the aircraft cannot reach their destinations.

\subsection{Algorithm modification}

To deal with the problem described in the previous section, the relative speed vector $\overrightarrow{v_{r}}$ is projected on the side of the cone even when it is in the circular part of the forbidden zone. In figure 6 aircraft $A$ and $B$ are moved to the left as soon as the relative speed $\overrightarrow{v_{r}}$ gets in the forbidden zone. This helps solve the conflict 


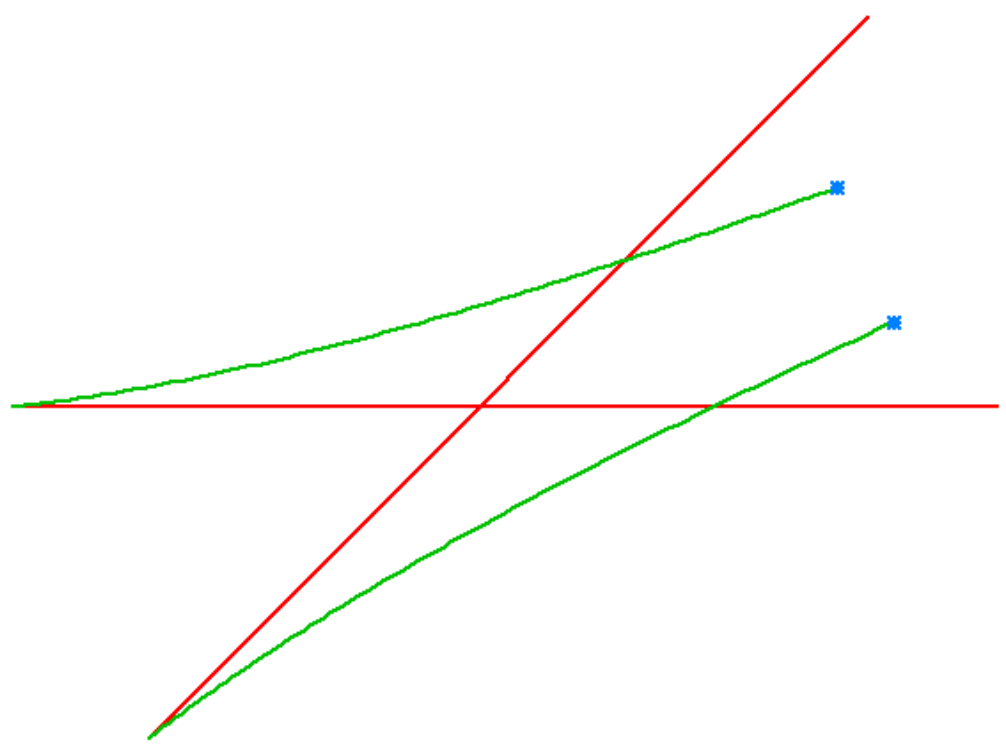

Figure 5: Two aircraft conflict with the original ORCA algorithm. Aircraft are turned opposite directions and fly parallel tracks without reaching their destinations. 


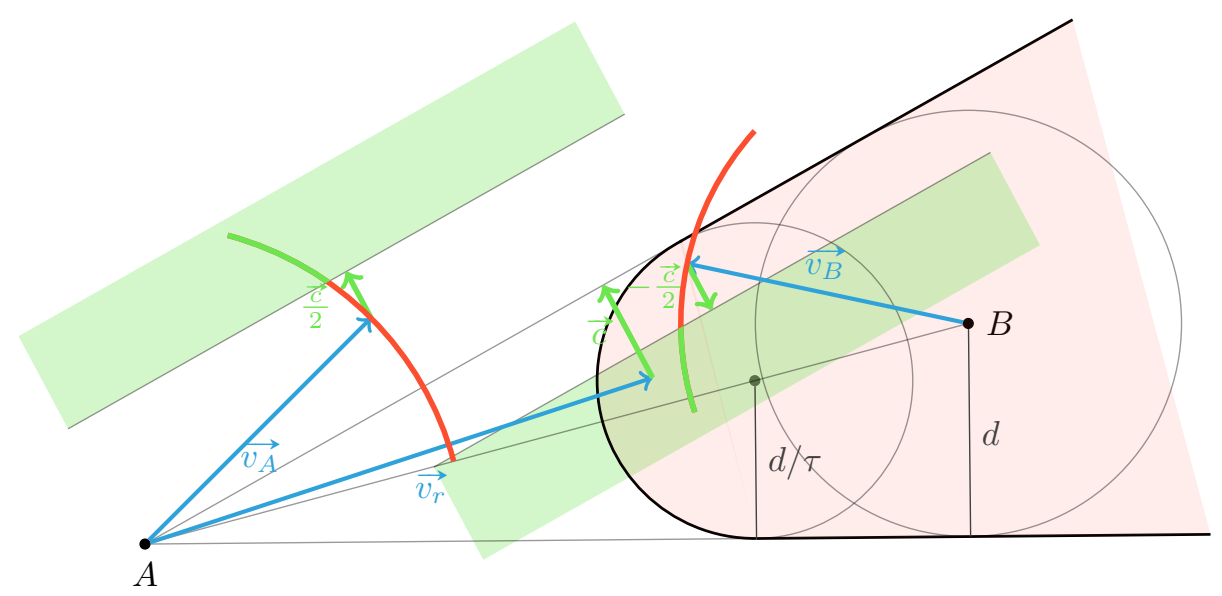

Figure 6: New model: the relative speed $\overrightarrow{v_{r}}$ is now projected on the closest side of the cone instead of the circular part.

and avoids the horizon effect described in the previous section.

In figure 7, the two aircraft converging to the center of the figure (red lines) with the same speed are turned left and can reach their destination while solving the conflict.

The effect of the algorithm can be observed on a bigger example involving 10 aircraft converging on a point from a circle arc. The converging angle between the aircraft is 6 degrees. They all fly with the same speed and are initially supposed to reach the center of the circle at the same time.

With the ORCA algorithm (see figure 8), the aircraft choose parallel tracks and cannot reach their initial destinations. With the CSORCA modification (see figure 99, the aircraft manage to solve their conflicts and reach their destinations.

\subsection{Comparison with an Add-Up strategy}

In the case of two aircraft conflict, the modified ORCA algorithm is very close to the Modified Voltage Potential (MVP) algorithm developed for the ASAS Hoekstra et al. (2002). The MVP algorithm, like CSORCA, uses the

full cone. The main difference between the modified ORCA and MVP appears for multiple aircraft conflicts. Instead of calculating the intersection of the 


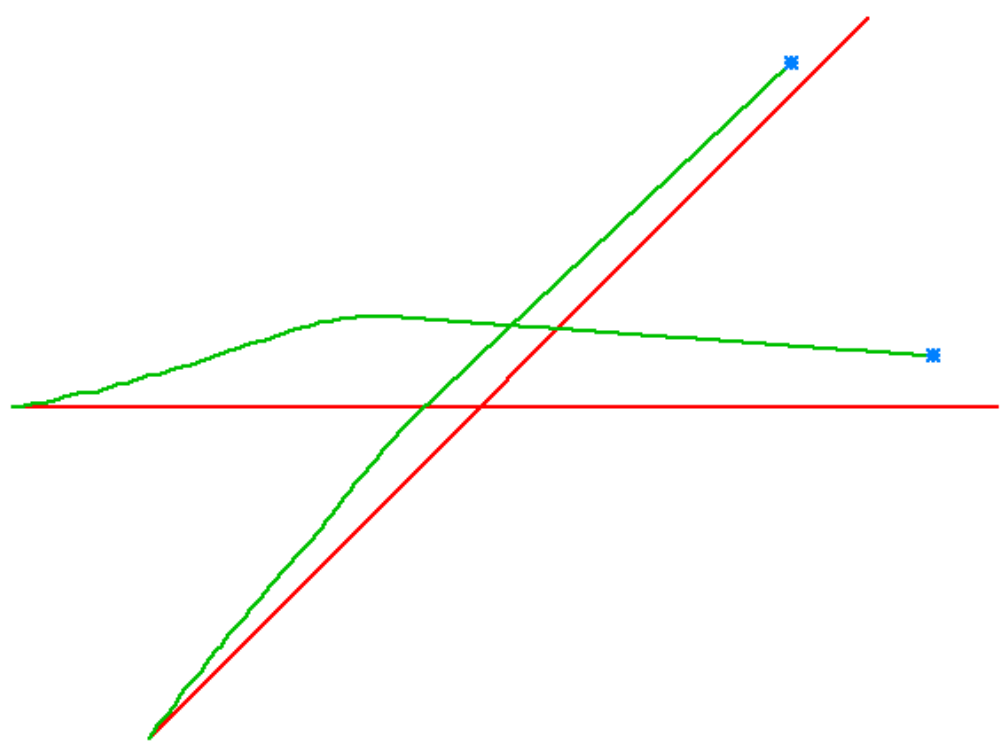

Figure 7: Two aircraft conflict with the modified ORCA algorithm. Both aircraft are turned left and the conflict is easily solved.

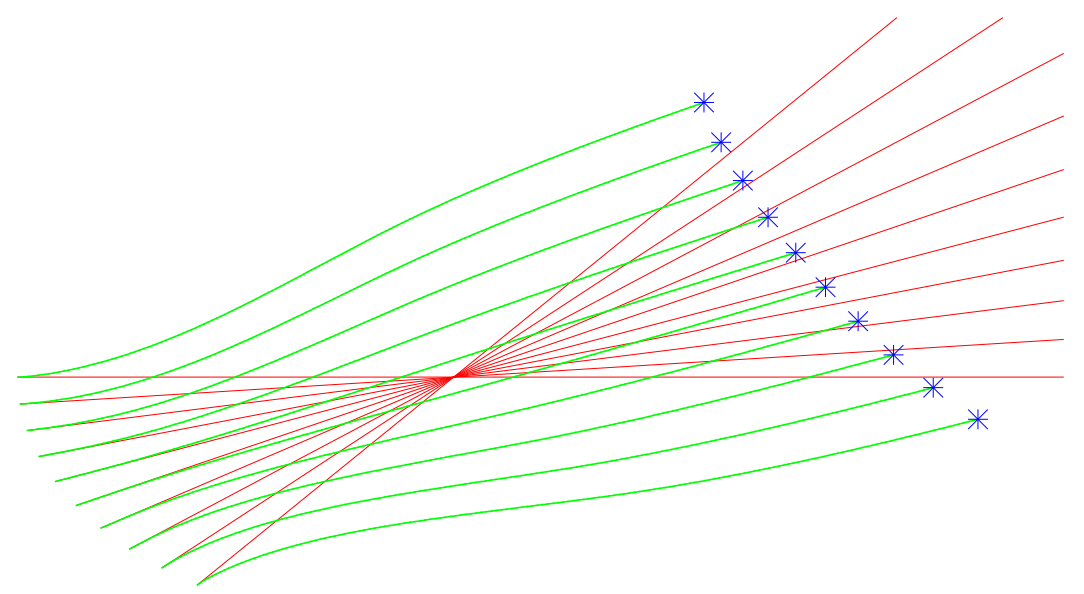

Figure 8: Ten aircraft conflict with the original ORCA algorithm. Aircraft choose parallel tracks. 


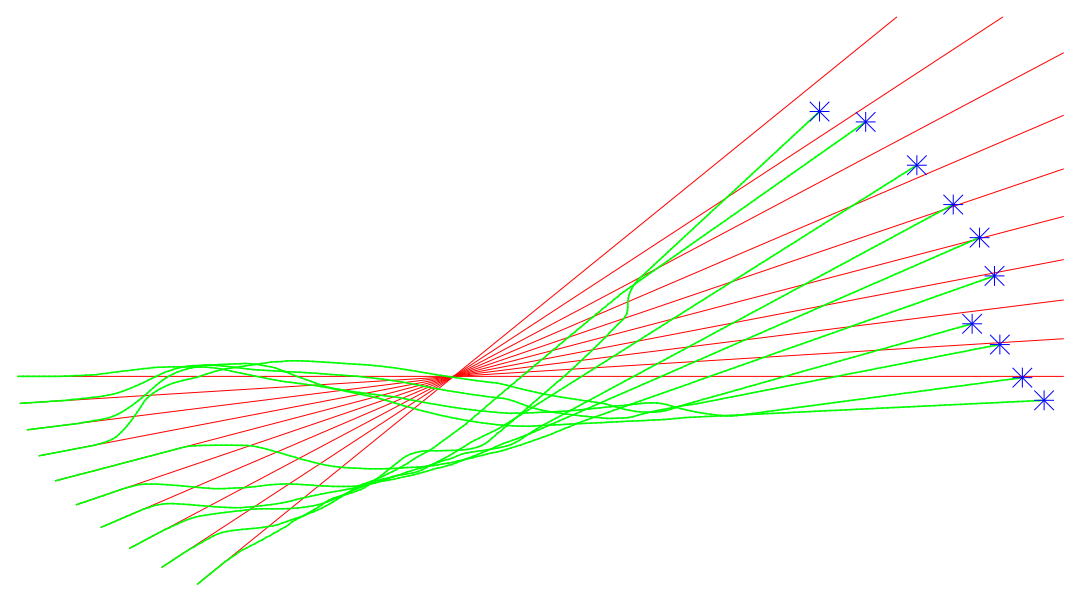

Figure 9: Ten aircraft conflict with the modified ORCA algorithm. Every aircraft reaches its destination without conflict.

semi-planes to define the conflict free heading domain, a speed deviation is calculated for each intruding aircraft and they are Added-Up to determine a resulting speed. When the speed norm is constrained, the heading changes are added-up and the resulting heading is limited by the maximum turning rate. This strategy is applied on a 8 aircraft conflict in figure 10. It cannot solve the 10 aircraft example without conflict. It will be called the Add-Up strategy in the next section.

\section{Experimental results}

We showed in Durand and Barnier (2015) that the ORCA algorithm in its initial version is not efficient when the speed norms are constrained. Even with low densities, such as presented in figure 11, the phenomenon presented in part 3.1 prevents aircraft from reaching their destination while staying in the window. In this figure, there are only ten aircraft flying at the same speed in a $500 \times 500$ nautical mile square area. With the original ORCA algorithm proposed by Van den Berg et al. van den Berg et al. (2011), small angle converging aircraft tend 


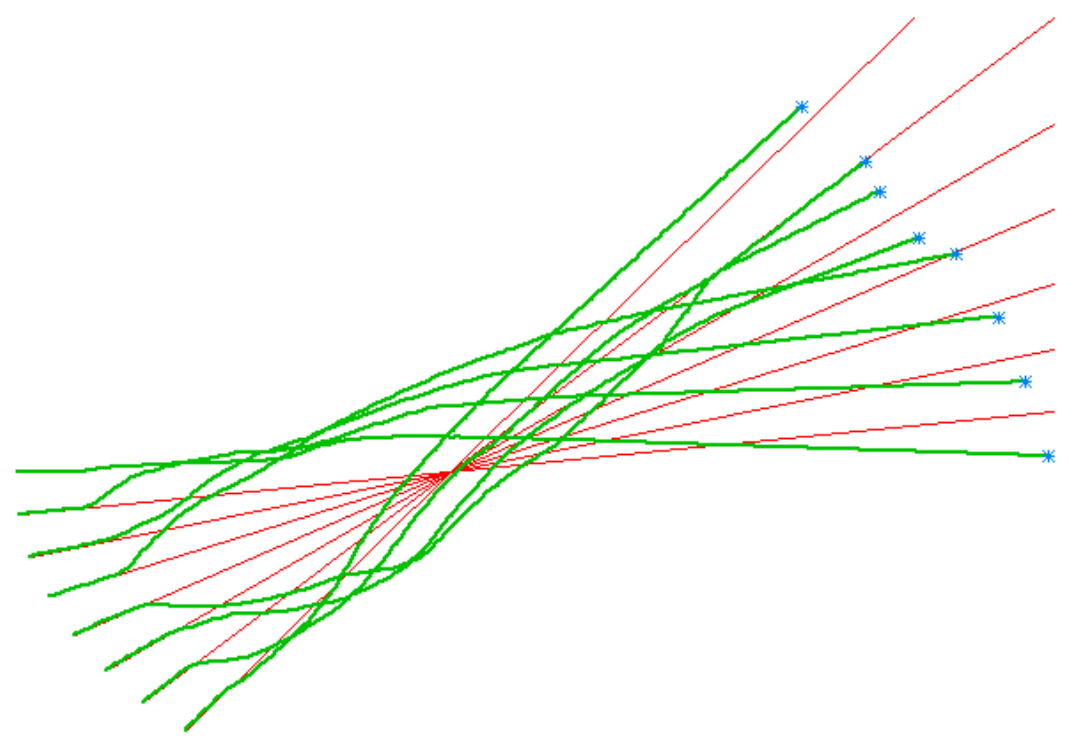

Figure 10: 8 aircraft conflict with the Add-Up Strategy. 


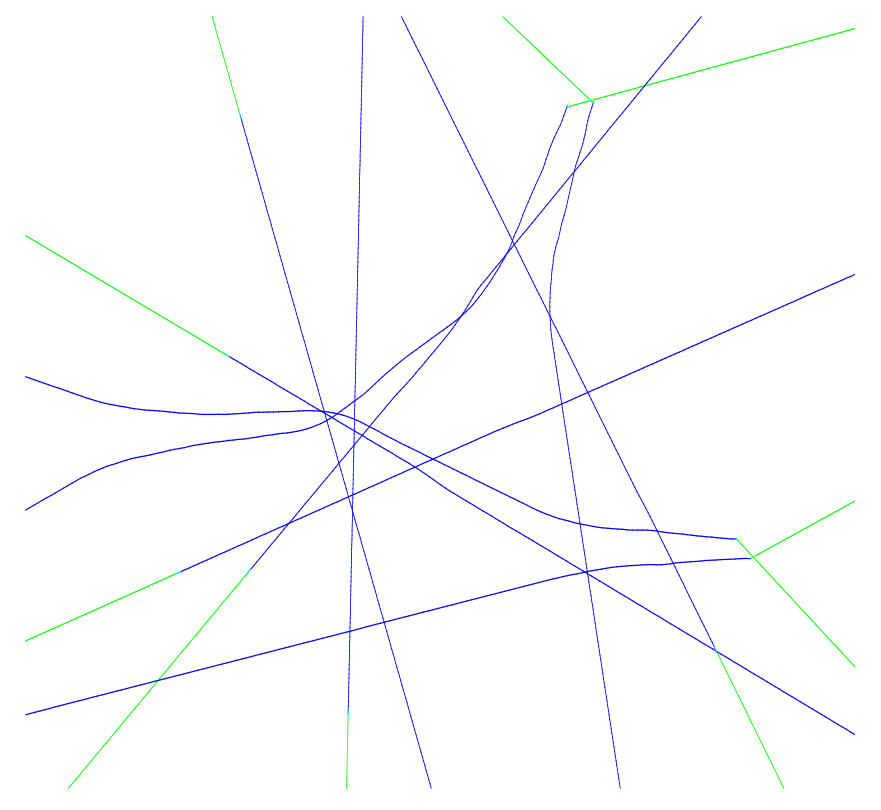

Figure 11: A scenario example with 10 aircraft and no speed norm modification.

to adopt parallel tracks that keep them away from their destination. Figure 12 represents a scenario where 130 aircraft fly in the same $500 \times 500$ nautical mile square area but can increase or reduce their speeds by $30 \%$. The original ORCA algorithm has no difficulty finding a solution for each of the 130 aircraft in this case.

In this section different scenarios are built to show how the modified version of the algorithm (CSORCA) behaves with different traffic densities and speed norm constraints.

4.1. ORCA and CSORCA comparison on various densities and constrained speed norms.

First the behaviors of ORCA and CSORCA are compared in a speed norm constrained environment to check how it handles different densities. Specifically, it is tested in a $500 \times 500$ nautical mile sector with varying numbers of aircraft 


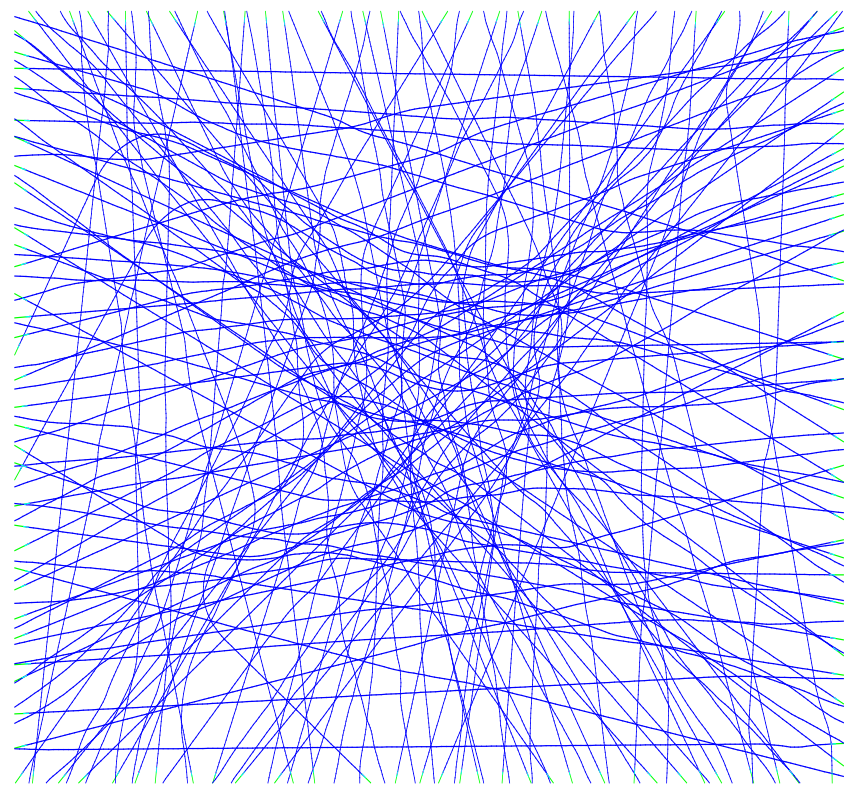

Figure 12: A scenario example with 130 aircraft and 30\% speed norm modification. 


\begin{tabular}{|c|c|c|c|c|c|c|c|c|c|c|c|}
\hline 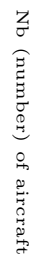 & 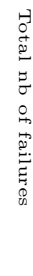 & $\begin{array}{l}z \\
z \\
0 \\
0 \\
\sigma \\
0 \\
0 \\
0 \\
0 \\
\vdots \\
\vdots \\
0 \\
0 \\
0 \\
0 \\
0\end{array}$ & 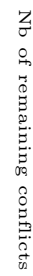 & 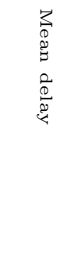 & 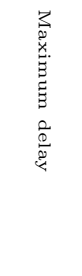 & $\begin{array}{l}\text { Z } \\
\sigma \\
\widehat{E} \\
E \\
5 \\
0 \\
0 \\
0 \\
0 \\
0 \\
0 \\
0 \\
0 \\
0 \\
0\end{array}$ & 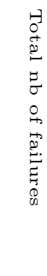 & $\begin{array}{l}z \\
\sigma \\
0 \\
0 \\
\sigma \\
0 \\
0 \\
0 \\
0 \\
\vdots \\
0 \\
0 \\
0 \\
0 \\
0 \\
0\end{array}$ & 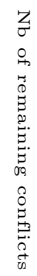 & 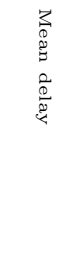 & 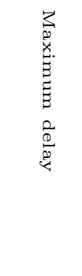 \\
\hline 10 & 4 & 1 & 3 & 1.00172 & 1.00763 & 10 & 96 & 96 & 0 & \multirow[t]{9}{*}{1.02100} & \multirow[t]{9}{*}{1.07680} \\
\hline 20 & 7 & 2 & 5 & 1.00477 & 1.01088 & 20 & 100 & 98 & 2 & & \\
\hline 30 & 26 & 3 & 23 & 1.01159 & 1.02739 & 30 & 100 & 93 & 7 & & \\
\hline 40 & 32 & 6 & 26 & 1.01979 & 1.03626 & 40 & 100 & 60 & 40 & & \\
\hline 50 & 53 & 14 & 39 & 1.02708 & 1.04028 & 50 & 100 & 25 & 75 & & \\
\hline 60 & 70 & 15 & 55 & 1.04270 & 1.06213 & 60 & 100 & 10 & 90 & & \\
\hline 70 & 81 & 28 & 53 & 1.05514 & 1.08631 & 70 & 100 & 7 & 93 & & \\
\hline 80 & 89 & 32 & 57 & 1.06571 & 1.10463 & 80 & 100 & 2 & 98 & & \\
\hline 90 & 99 & 20 & 79 & 1.07024 & 1.07024 & 90 & 100 & 1 & 99 & & \\
\hline
\end{tabular}

Table 1: Total number of failures, due to border violations or remaining conflicts, mean and max delays for 10 to 90 aircraft with no speed norm variation and 3 degrees/second maximum turning rate. Left: CSORCA - Right: ORCA

$n$ and different parameters for the turning angle. In the following, low density is defined as 10, 20, 30 aircraft, medium as 40,50 or 60 aircraft, and high as 70, 80 or 90 aircraft. The density of conflicts is proportional to the square of the density of traffic because the traffic is randomly distributed.

The aircraft speeds are set to 1 (speeds are normalized), which means that all aircraft fly at the same speed. This is not totally realistic, but similar results are obtained with nominal speed norms varying by $\pm 20 \%$ and some preliminary tests have shown that the worst situations appear when speed norms are close to each other. The time step $t_{s}$ is set to 3 seconds.

The parameter $\tau$ is fixed at $\tau=120 \mathrm{~s}$ (i.e. 2 minutes) because it is a good 315 compromise: if the lookahead time is too long, the algorithm is less efficient for high densities because too many conflicts are simultaneously taken into account and the permitted zones often are empty; whereas if $\tau$ is too short, some conflicts appear too late to be resolved. A sensitivity analysis done with a 3 degrees/second maximum turn rate and no speed norm variation showed that for low densities (up to 30 aircraft) a longer $\tau$ value can minimize the number of failures (see table 2 but for higher densities, $\tau=120$ seconds is the best 


\begin{tabular}{|c|c|c|c|c|c|}
\hline 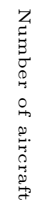 & $\begin{array}{l}y \\
11 \\
0 \\
0 \\
0 \\
0 \\
0 \\
0 \\
0 \\
0\end{array}$ & $\begin{array}{l}-1 \\
\| 1 \\
0 \\
0 \\
\text { dn } \\
0 \\
\delta \\
0 \\
0\end{array}$ & $\begin{array}{l}-1 \\
\text { II } \\
\text { N } \\
0 \\
0 \\
0 \\
0 \\
0 \\
0 \\
0 \\
0\end{array}$ & $\begin{array}{l}-1 \\
\| 1 \\
\| \\
5 \\
0 \\
0 \\
0 \\
0 \\
0 \\
0 \\
0 \\
0 \\
0\end{array}$ & $\begin{array}{l}-1 \\
\| \\
\sim \\
\infty \\
0 \\
0 \\
0 \\
0 \\
0 \\
0 \\
0 \\
0\end{array}$ \\
\hline 10 & 10 & 7 & 4 & 3 & 2 \\
\hline 20 & 31 & 24 & 7 & 15 & 10 \\
\hline 30 & 71 & 48 & 26 & 23 & 16 \\
\hline 40 & 79 & 60 & 32 & 34 & 50 \\
\hline 50 & 94 & 76 & 53 & 67 & 81 \\
\hline 60 & 98 & 79 & 70 & 82 & 97 \\
\hline 70 & 100 & 85 & 81 & 99 & 100 \\
\hline 80 & 100 & 91 & 89 & 100 & 100 \\
\hline 90 & 100 & 100 & 99 & 100 & 100 \\
\hline
\end{tabular}

Table 2: Total number of failures, for different values of $\tau$ for 10 to 90 aircraft with no speed norm variation and 3 degrees/second maximum turning rate. CSORCA

compromise.

100 runs are first executed for different values of $n$ and a maximum standard turning rate of 3 degrees per second. The speed norms are strictly constrained.

For each execution, the origins and destinations of the aircraft are randomly chosen on the edges of the square. The aircraft are separated by at least 3 separation standards (15 NM) at the origin and the destination. A quarter of the traffic goes from the east side to the west side, another quarter from west to east, a quarter from north to south and the last one from south to north.

For each scenario, the number of runs which fail because aircraft are "pushed" out of the $500 \times 500$ nautical mile sector before reaching their destination is counted. The number of runs which do not solve every conflict is also counted. For the remaining runs, the trajectory lengthening (the ratio of the modified trajectory and the original trajectory) is measured.

Table 1 gives the experimental results for densities varying from 10 to 90 aircraft with the CSORCA (left) and ORCA (right) algorithm, no speed norm change and a maximum 3 degrees per second turning rate.

The CSORCA algorithm helps solve more conflicts in denser airspace. For $n=40$ the number of failures is still close to zero with the CSORCA algorithm whereas it is already a $100 \%$ for 20 aircraft with ORCA algorithm. With 


\begin{tabular}{|c|c|c|c|c|c|}
\hline $\begin{array}{l}Z \\
\sigma \\
\widehat{\Xi} \\
5 \\
5 \\
0 \\
0 \\
0 \\
0 \\
0 \\
0 \\
0 \\
0 \\
0 \\
0\end{array}$ & 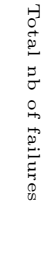 & 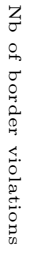 & 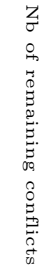 & 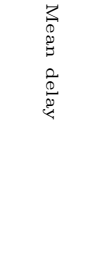 & 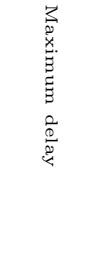 \\
\hline 10 & 15 & 1 & 14 & 1.001428 & 1.03708 \\
\hline 20 & 81 & 0 & 81 & 1.002502 & 1.003631 \\
\hline 30 & 100 & 0 & 100 & & \\
\hline 40 & 100 & 0 & 100 & & \\
\hline 50 & 100 & 0 & 100 & & \\
\hline 60 & 100 & 0 & 100 & & \\
\hline 70 & 100 & 0 & 100 & & \\
\hline 80 & 100 & 0 & 100 & & \\
\hline 90 & 100 & 0 & 100 & & \\
\hline
\end{tabular}

Table 3: Add-Up Strategy: Total number of failures, due to border violations or remaining conflicts, mean and max delays for 10 to 90 aircraft with no speed norm variation and 3 degrees/second maximum turning rate.

CSORCA, the number of failures grows with the density and reaches $100 \%$ only for high densities involving 90 aircraft.

The Add-Up strategy (see figure 3) is better than the initial ORCA approach but it is unable to solve 30 aircraft problems. The main cause off failure is due to a separation violation for multiple aircraft conflicts.

When doubling the maximum turning rate by allowing a maximum 6 degrees per second turning rate, table 4 shows that the number of failures is not reduced except for very low densities for which it drops from 96 to 93 . However, the CSORCA algorithm gives much better results, with almost no failures before reaching 50 aircraft and a lower rate of failures for higher densities.

When comparing the results in terms of border violation, tables 1 and 4 show that for the ORCA algorithm and low densities, the main reason for failure is due to border violation. This is a consequence of the phenomenon observed in figure 6 where aircraft tend to "push" each other and finally reach the limits of the resolution area. When the density increases the number of failures due to separation standard violation increases as well: before reaching the border of the resolution area, aircraft may encounter other trajectories that can lead to a 


\begin{tabular}{|c|c|c|c|c|c|c|c|c|c|c|c|}
\hline 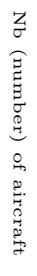 & 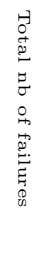 & $\begin{array}{l}\mathrm{z} \\
0 \\
0 \\
0 \\
\sigma \\
0 \\
0 \\
0 \\
0 \\
\vdots \\
\vdots \\
0 \\
0 \\
0 \\
0 \\
0 \\
0\end{array}$ & 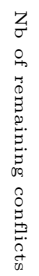 & 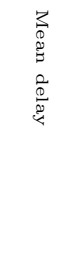 & 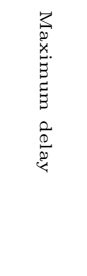 & 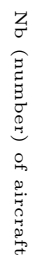 & 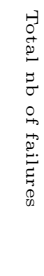 & $\begin{array}{l}z \\
z \\
0 \\
0 \\
\sigma \\
0 \\
0 \\
0 \\
0 \\
\vdots \\
\vdots \\
0 \\
0 \\
0 \\
0 \\
0 \\
0\end{array}$ & $\begin{array}{l}z \\
z \\
0 \\
0 \\
0 \\
0 \\
5 \\
0 \\
0 \\
0 \\
0 \\
0 \\
0 \\
0 \\
0 \\
0 \\
0\end{array}$ & $\begin{array}{l}3 \\
0 \\
0 \\
0 \\
0 \\
0 \\
0 \\
2 \\
2\end{array}$ & 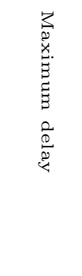 \\
\hline 10 & 0 & 0 & 0 & 1.00228 & 1.01115 & 10 & 93 & 93 & 0 & \multirow[t]{9}{*}{1.07941} & \multirow[t]{9}{*}{1.17754} \\
\hline 20 & 1 & 0 & 1 & 1.00537 & 1.01479 & 20 & 100 & 99 & 1 & & \\
\hline 30 & 4 & 0 & 4 & 1.01299 & 1.03417 & 30 & 100 & 89 & 11 & & \\
\hline 40 & 2 & 0 & 2 & 1.02099 & 1.03659 & 40 & 100 & 49 & 51 & & \\
\hline 50 & 14 & 3 & 11 & 1.03308 & 1.05832 & 50 & 100 & 21 & 79 & & \\
\hline 60 & 23 & 4 & 19 & 1.04661 & 1.07188 & 60 & 100 & 15 & 85 & & \\
\hline 70 & 36 & 7 & 29 & 1.06012 & 1.08392 & 70 & 100 & 3 & 97 & & \\
\hline 80 & 53 & 21 & 32 & 1.07471 & 1.11324 & 80 & 100 & 1 & 99 & & \\
\hline 90 & 74 & 21 & 53 & 1.09584 & 1.12779 & 90 & 100 & 0 & 100 & & \\
\hline
\end{tabular}

Table 4: Total number of failures, due to border violations or remaining conflicts, mean and max delays for 10 to 90 aircraft with no speed norm variation and 6 degrees/second maximum turning rate. Left: CSORCA - Right: ORCA

separation standard violation. For the CSORCA algorithm, both the number of border violations and remaining conflicts increase with the density even though the number of failures is lower when the maximum tuning rate is doubled. The mean delay due to maneuvers increases with the density, which seems logical and when the maximum turning rate is increased, which was also expected.

\subsection{ORCA and CSORCA comparison on various densities and small speed norm variations.}

In this section the previous tests are reproduced and allow a small speed norm variation to check the behavior of the modified algorithm when the speed norm constraint is relaxed. A $5 \%$ speed norm modification around the nominal speed is allowed (see figure 5). With the CSORCA algorithm the number of failures is decreased, even for high densities. With the ORCA algorithm, the number of

failures is only significantly decreased for very low densities (10 aircraft). This shows that the modification made on ORCA is compatible with a relaxed speed norm constraint environment.

When the speed norm can be modified by $+-15 \%$, the CSORCA algorithm 


\begin{tabular}{|c|c|c|c|c|c|c|c|c|c|c|c|}
\hline 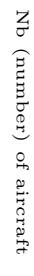 & 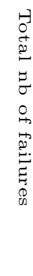 & $\begin{array}{l}z \\
z \\
0 \\
0 \\
\sigma \\
0 \\
0 \\
0 \\
0 \\
\vdots \\
\vdots \\
0 \\
0 \\
0 \\
0 \\
0\end{array}$ & 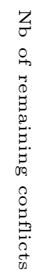 & 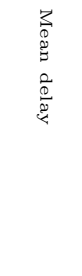 & 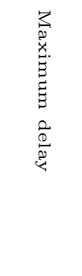 & $\begin{array}{l}\text { Z } \\
\sigma \\
\widehat{E} \\
E \\
5 \\
0 \\
0 \\
0 \\
0 \\
0 \\
0 \\
0 \\
0 \\
0 \\
0\end{array}$ & 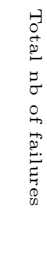 & $\begin{array}{l}z \\
\sigma \\
0 \\
i \\
\sigma \\
0 \\
0 \\
0 \\
0 \\
\vdots \\
0 \\
0 \\
0 \\
0 \\
0 \\
0 \\
0\end{array}$ & 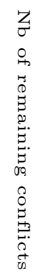 & 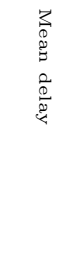 & 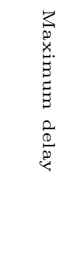 \\
\hline 10 & 0 & 0 & 0 & 1.00061 & 1.00331 & 10 & 72 & 72 & 0 & 1.00666 & 1.02857 \\
\hline 20 & 0 & 0 & 0 & 1.00168 & 1.00730 & 20 & 99 & 99 & 0 & 1.07514 & 1.07514 \\
\hline 30 & 0 & 0 & 0 & 1.00382 & 1.00969 & 30 & 97 & 97 & 0 & 1.03329 & 1.04360 \\
\hline 40 & 1 & 0 & 1 & 1.00664 & 1.01412 & 40 & 100 & 99 & 1 & & \\
\hline 50 & 4 & 2 & 2 & 1.01179 & 1.02745 & 50 & 100 & 85 & 15 & & \\
\hline 60 & 14 & 2 & 12 & 1.01634 & 1.03171 & 60 & 100 & 61 & 39 & & \\
\hline 70 & 12 & 4 & 8 & 1.02170 & 1.03884 & 70 & 100 & 38 & 62 & & \\
\hline 80 & 20 & 5 & 15 & 1.03050 & 1.05314 & 80 & 100 & 9 & 91 & & \\
\hline 90 & 47 & 22 & 25 & 1.03485 & 1.04844 & 90 & 100 & 4 & 96 & & \\
\hline
\end{tabular}

Table 5: Total number of failures, due to border violations or remaining conflicts, mean and max delays for 10 to 90 aircraft with $+-5 \%$ maximum speed norm variation and 3 degrees/second maximum turning rate. Left: CSORCA - Right: ORCA

never fails for densities up to 60 aircraft (see figure 6) and even with high densities (90 aircraft), the rate of failure is only 10\%. With the ORCA algorithm, there is still more than $20 \%$ of failure for only 10 aircraft and no solution is found for high densities.

\section{Conclusion and Future Work}

The ORCA algorithm proposed by Vandenberg et al. van den Berg et al. (2011) is very efficient for solving conflicts between aircraft that can easily modify their speed ranges. It is a very effective geometric self-separation algorithm that can safely handle complex situations. When several aircraft are involved in a conflict, the projection of the relative speed outside the conflicting area ensures conflict free trajectories for every robot. The more maneuverable a robot is, the more options it has to escape the conflicting zone. In the worst cases it can be completely stopped by the ORCA algorithm at its current position to wait for the rest of the traffic to clear the area. This algorithm could be a good candidate for aircraft separation because it ensures a solution for multiple robot conflicts provided that the aircraft are sufficiently maneuverable. The issues 


\begin{tabular}{|c|c|c|c|c|c|c|c|c|c|c|c|}
\hline 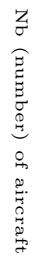 & 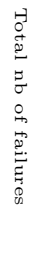 & $\begin{array}{l}z \\
\sigma \\
0 \\
0 \\
\sigma \\
0 \\
0 \\
0 \\
0 \\
0 \\
\vdots \\
\vdots \\
0 \\
0 \\
0 \\
0 \\
0 \\
0\end{array}$ & 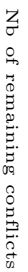 & 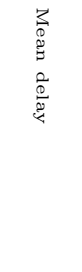 & 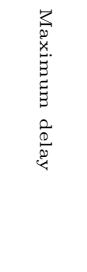 & 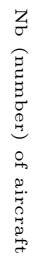 & 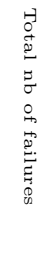 & $\begin{array}{l}z \\
z \\
0 \\
0 \\
\sigma \\
0 \\
0 \\
0 \\
0 \\
\vdots \\
\vdots \\
0 \\
0 \\
0 \\
0 \\
0\end{array}$ & 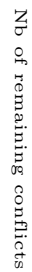 & 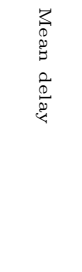 & 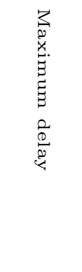 \\
\hline 10 & 0 & 0 & 0 & 1.00019 & 1.00072 & 10 & 21 & 21 & 0 & 1.00166 & 1.02927 \\
\hline 20 & 0 & 0 & 0 & 1.00070 & 1.00223 & 20 & 50 & 50 & 0 & 1.00657 & 1.04444 \\
\hline 30 & 0 & 0 & 0 & 1.00162 & 1.00435 & 30 & 79 & 79 & 0 & 1.01374 & 1.03287 \\
\hline 40 & 0 & 0 & 0 & 1.00294 & 1.00842 & 40 & 92 & 92 & 0 & 1.02868 & 1.04121 \\
\hline 50 & 0 & 0 & 0 & 1.00525 & 1.01143 & 50 & 97 & 97 & 0 & 1.02558 & 1.03069 \\
\hline 60 & 0 & 0 & 0 & 1.00721 & 1.01284 & 60 & 100 & 100 & 0 & & \\
\hline 70 & 1 & 1 & 0 & 1.01070 & 1.02259 & 70 & 100 & 100 & 0 & & \\
\hline 80 & 5 & 3 & 2 & 1.01472 & 1.02449 & 80 & 100 & 89 & 11 & & \\
\hline 90 & 10 & 5 & 5 & 1.01791 & 1.03095 & 90 & 100 & 67 & 33 & & \\
\hline
\end{tabular}

Table 6: Total number of failures, due to border violations or remaining conflicts, mean and max delays for 10 to 90 aircraft with no $+-15 \%$ maximum speed norm variation and 3 degrees/second maximum turning rate. Left: CSORCA - Right: ORCA

of the ORCA algorithm in the air traffic control context were first exhibited by Durand and Barnier in Durand and Barnier (2015). We showed that the speed constraint prevented the algorithm from solving conflicts when aircraft had similar constrained speeds. When the speed is constrained, the algorithm loses its performance even for low densities. When aircraft with similar speeds converge with small angles they tend to choose parallel tracks that keep them away from their destination. The algorithm enhancement proposed in this article aims at solving this issue. By projecting the relative speed outside the conflicting cone as soon as the vector enters the forbidden area, aircraft tend to make coordinated maneuvers that solve conflicts instead of delaying their resolutions. An example of two aircraft was introduced to illustrate the behavior of the original ORCA algorithm and the CSORCA enhancement. A 10 aircraft example was then introduced: the 10 aircraft converged with a 6 degree angle to the center of a circle at the same speed. The CSORCA algorithm manages to solve every conflict whereas the ORCA approach only delays the resolution by giving parallel tracks to every aircraft. An Add-Up strategy was compared to the CSORCA algorithm but it was only able to deal with 8 aircraft. 
ORCA and CSORCA approaches were then compared on random traffic scenarios with various densities and different turning rates. With no speed norm variation and a standard turning rate of 3 degrees per second, ORCA can rarely handle 10 aircraft whereas CSORCA can still manage $47 \%$ of the scenarios involving 70 aircraft. The Add-Up strategy gave better results than the ORCA approach but only for low densities (less than 30 aircraft).

With a 6 degree per second turning rate, ORCA does not behave any better whereas CSORCA can handle $53 \%$ of the scenarios involving 90 aircraft. Relaxing the speed norm by $\pm 5 \%$ slightly improves results with ORCA: 10 aircraft examples are solved in $28 \%$ of the scenarios with a 3 degree turning rate. With CSORCA, $80 \%$ of the scenarios involving 80 aircraft ares solved. Relaxing the speed norm by $\pm 15 \%$ improves results with ORCA even more: $50 \%$ of the scenarios involving 20 aircraft are solved with a 3 degree turning rate. With CSORCA, $90 \%$ of the scenarios involving 90 aircraft are solved. These statistical results illustrate the efficiency of the modification made on the initial ORCA algorithm.

The next step will be to add vertical maneuvers to the model and check the behavior of both ORCA and CSORCA when a vertical option is added to maneuvers. The vertical option will not be combined with a horizontal maneuver in order to comply with current practices in air traffic control. The impact of errors on the information captured by the aircraft should also be carefully studied. Allignol et al. Allignol et al. (2017) have conducted many experiments for a single UAS interfering with real traffic. Results show that performances are reduced. It would be interesting to adapt the ORCA and CSORCA approaches to take errors into account.

\section{References}

Alam, S., Shafi, K., Abbass, H.A., Barlow, M., 2009. An ensemble approach for conflict detection in free flight by data mining. Transportation Research Part C: Emerging Technologies 17, $298-317$. 
Allignol, C., Barnier, N., Durand, N., Alliot, J.M., 2013. A new framework for solving en-route conflicts, in: Proceedings of the 10th ATM R\&D Seminar, Chicago, IL.

Allignol, C., Barnier, N., Durand, N., Manfredi, G., Blond, É., 2017. Assessing the Robustness of a UAS Detect \& Avoid Algorithm, in: 12th USA/Europe Air Traffic Management Research and Development Seminar, Seattle, United States.

Alonso-Ayuso, A., Escudero, L.F., Martin-Campo, F.J., 2016. Exact and approximate solving of the aircraft collision resolution problem via turn changes. Transportation Science 50, 263-274. https://doi.org/10.1287/trsc.2014.0557.

Archambault, N., Durand, N., 2004. Scheduling heuristics for on-board sequential air conflict solving, in: 23rd Digital Avionics Systems Conference, AIAA. IEEE, Salt Lake City, UT. pp. 3.1-9.

Balasooriyan, S., 2017. Multi-aircraft Conflict Resolution using Velocity Obstacles. Master's thesis. TUDelft.

van den Berg, J., Guy, S.J., Lin, M., Manocha, D., 2011. Reciprocal n-body collision avoidance, in: Pradalier, C., Siegwart, R., Hirzinger, G. (Eds.), Robotics Research: The 14th International Symposium ISRR, Springer, Berlin, Heidelberg. pp. 3-19.

van den Berg, J., Lin, M., Manocha, D., 2008. Reciprocal velocity obstacles for real-time multi-agent navigation, in: 2008 IEEE International Conference on Robotics and Automation, pp. 1928-1935.

Bilimoria, K., 2000. A geometric optimization approach to aircraft conflict resolution, in: Proceedings of the 18th Applied Aerodynamics Conference.

Bonini, D., Dupré, C., Granger, G., 2009. How erasmus can support an increase in capacity in 2020, in: Proceedings of the 7th International Conference on Computing, Communications and Control Technologies: CCCT 2009, Orlando, Florida. 
Bulusu, V., Sengupta, R., Liu, Z., 2016. Unmanned aviation: To be free or not to be free? a complexity based approach, in: 7th International Conference on Research in Air Transportation, Drexel University, Philadelphia, PA.

Chiang, Y.J., Klosowski, J.T., Lee, C., Mitchell, J.S., 1997. Geometric algorithms for conflict detection/resolution in air traffic management, in: Proceedings of the 36th Conference on Decision and Control, San Diego, CA. pp. 1835-1840.

Christodoulou, M.A., Kontogeorgou, C., 2008. Collision avoidance in commercial aircraft free flight via neural networks and non-linear programming. International Journal of Neural Systems 18, 371-387.

D. Bilimoria, K., Sridhar, B., B. Chatterji, G., Sh eth, K., R. Grabbe, S., 2001. Facet: Future atm concepts evaluation tool. Air Traffic Control Quarterly 9, 1-20.

d'Engelbronner, J., Borst, C., Ellerbroek, J., Van Paassen, M.M., Mulder, M., 2015. Solution-space-based analysis of dynamic air traffic controller workload. Journal of Aircraft , 1146-1161.

Durand, N., Alliot, J.M., 1997. Optimal resolution of en route conflicts, in: Proceedings of the 1st ATM R\&D Seminar, Saclay, France.

Durand, N., Alliot, J.M., Frédéric, M., 2000. Neural nets trained by genetic algorithms for collision avoidance. Applied Intelligence 13, 205-213.

Durand, N., Alliot, J.M., Noailles, J., 1996. Automatic aircraft conflict resolution using genetic algorithms, in: 11th Annual Symposium on Applied Computing, ACM, Philadelphia, PA. pp. 289-298.

Durand, N., Barnier, N., 2015. Does atm need centralized coordination? autonomous conflict resolution analysis in a constrained speed environment, in: 11th USA/Europe Air Traffic Management Research and Developpment Seminar. 
Eby, M.S., Kelly, III, W.E., 1999. Free flight separation assurance using distributed algorithms, in: IEEE Aerospace Conference. Proceedings, pp. 429-441.

Emmanuel Sunil, J.E., Hoekstra, J.M., 2018. Camda: Capacity assessment method for decentralized air traffic control, in: 8th International Conference on Research in Air Transportation, UPC, Castelldelfels, Spain.

${ }_{495}$ Frazzoli, E., Mao, Z.H., Oh, J.H., Feron, E., 2001. Resolution of conflicts involving many aircraft via semidefinite programming. Journal of Guidance, Control and Dynamics 24, 79-86.

Gariel, M., Feron, E., 2009. 3d conflict avoidance under uncertainties, in: 28th Digital Avionics Systems Conference, AIAA. IEEE, Orlando, FL. pp. 4.E.3-1-4.E.3-8.

Granger, G., Durand, N., Alliot, J.M., 2001a. Optimal resolution of en-route conflicts, in: Proceedings of the 4th ATM R\&D Seminar, Santa Fe, NM.

Granger, G., Durand, N., Alliot, J.M., 2001b. Token allocation strategy for free-flight conflict solving, in: Hirsh, H., Chien, S. (Eds.), IAAI 2001, 13th Conference on Innovative Applications of Artificial Intelligence, AAAI Press, Seattle, WA. pp. 59-64.

Hoekstra, J., van Gent, R., Ruigrok, R., 2002. Designing for safety: the 'free flight' air traffic management concept. Reliability Engineering \& System Safety $75,215-232$.

Hu, J., Prandini, M., Nilim, A., Sastry, S., 2002. Optimal coordinated maneuvers for three dimensional aircraft conflict resolution. Journal of Guidance, Control and Dynamics 25, 888-900.

Hwang, I., J.Kim, Tomlin, C., 2007. Protocol-based conflict resolution for air traffic control. ATC Quarterly 15, 1-34.

515 Košecká, J., Tomlin, C., Pappas, G.J., Sastry, S., 1998. 2 1/2 d conflict resolution maneuvers for atms, in: Proceedings of the 37th IEEE Conference on Decision and Control, pp. 2650-2655. 
Krella, F., et al., 1989. Arc 2000 scenario (version 4.3). Technical Report. Eurocontrol.

Le Ny, J., Pappas, G.J., 2010. Geometric programming and mechanism design for air traffic conflict resolution, in: Proceedings of the 2010 American Control Conference (ACC), pp. 3069-3074.

Lehouillier, T., Omer, J., Soumis, F., Desaulniers, G., 2017. Two decomposition algorithms for solving a minimum weight maximum clique model for the air conflict resolution problem. European Journal of Operational Research 256, $696-712$.

Oh, J.H., Shewchun, J.M., Feron, E., 1997. Design and analysis of conflict resolution algorithms via positive semidefinite programming [aircraft conflict resolution], in: Proceedings of the 36th Conference on Decision and Control, San Diego, CA. pp. 4179-4185.

Omer, J., Farges, J.L., 2013. Hybridization of nonlinear and mixed-integer linear programming for aircraft separation with trajectory recovery. IEEE Transactions on Intelligent Transportation Systems 14, 1218-1230.

Pallottino, L., Bicchi, A., Feron, E., 2001. Mixed integer programming for aircraft conflict resolution, in: AIAA Guidance, Navigation, and Control Conference and Exhibit, Montrã $(a)$ al, Canada.

Pallottino, L., Feron, E., Bicchi, A., 2002. Conflict resolution problems for air traffic management systems solved with mixed integer programming. IEEE Transactions on Intelligent Transportation Systems 3, 3-11. cooperative policy for conflict resolution in multivehicle systems. Trans. Rob. $23,1170-1183$.

Peyronne, C., Conn, A., Mongeau, M., Delahaye, D., 2015. Solving air-traffic conflict problems via local continuous optimization. European Journal of Operational Research 241, 502-512. 
Rey, D., Hijazi, H., 2017. Complex number formulation and convex relaxations for aircraft conflict resolution, in: 2017 IEEE 56th Annual Conference on Decision and Control (CDC), Melbourne, Australia. pp. 88-93.

Ruigrok, R.C., Hoekstra, J.M., 2007. Human factors evaluations of free flight: Issues solved and issues remaining. Applied Ergonomics 38, 437 - 455. Flightdeck of the Future.

Schouwenaars, T., Feron, E., 2004. Decentralized cooperative trajectory planning of multiple aircraft with hard safety guarantees, in: AIAA Guidance, Navigation, and Control Conference and Exhibit.

Snape, J., Manocha, D., 2010. Navigating multiple simple-airplanes in 3D workspace, in: IEEE International Conference on Robotics and Automation (ICRA), Anchorage, AK. pp. 3974-3980.

Sunil, E., Ellerbroek, J., Hoekstra, J., Maas, J., 2017. Modeling airspace stability and capacity for decentralized separation, in: 12th USA/Europe Air Traffic Management Research and Development Seminar, Seattle, United States.

Zeghal, K., 1998. A comparison of different approaches based on force fields for coordination among multiple mobiles, in: Proceedings. IEEE/RSJ International Conference on Intelligent Robots and Systems. Innovations in Theory, Practice and Applications, pp. 273-278. 